\title{
ON DUAL BANACH ALGEBRAS
}

\author{
PAK-KEN WONG
}

(Communicated by Palle E. T. Jorgensen)

\begin{abstract}
Let $A$ be a semisimple Banach algebra with $\|\cdot\|$, which is a dense subalgebra of a semisimple Banach algebra $B$ with $|\cdot|$ such that $\|\cdot\|$ majorizes $|\cdot|$ on $A$. The purpose of this paper is to investigate the dual property between the algebras $A$ and $B$. Some well-known results follow from this paper.
\end{abstract}

\section{INTRODUCTION}

Let $A$ be a semisimple Banach algebra with norm $\|\cdot\|$ which is a dense subalgebra of a semisimple Banach algebra $B$ with $|\cdot|$ such that $\|\cdot\|$ majorizes $|\cdot|$ on $A$. The purpose of this paper is to investigate the dual property between the algebras $A$ and $B$.

It is shown that if $A$ is a dual algebra, then $B$ is a dual algebra if and only if, $R=\operatorname{cl}_{B}(R \cap A)$, for any proper closed right (left) ideal $R$ of $B$. On the other hand, if $B$ is a dual algebra, then $A$ is a dual algebra if and only, for any proper closed right (left) ideal $N$ of $A, N=c l_{B}(N) \cap A$ and for any proper closed right (left) ideal $R$ of $B, R=\operatorname{cl}_{B}(R \cap A)$. If $A$ is a two-sided ideal of $B$ and $B$ has a bounded right approximate identity and a bounded left approximate identity, then we show that $A$ is a dual algebra if and only if $B$ is a dual algebra and $x \in c_{A}(x A) \cap c_{A}(A x)$ for all $x$ in $A$. Some well-known results follow from our results.

\section{NOTATION AND PRELIMINARIES}

Definitions not explicitly given are taken from Rickart [5].

Let $A$ be a Banach algebra. For any subset $E$ of $A$, let $c l_{A}(E)$ denote the closure of $E$ in $A$ and $\ell_{A}(E)$ (resp. $r_{A}(E)$ ) the left (resp. right) annihilator of $E$ in $A$. Then $A$ is called an annihilator algebra if $\ell_{A}(A)=r_{A}(A)=(0)$ and if for every proper closed right ideal $I$ and every proper closed left ideal $J$ $\ell_{A}(I) \neq(0)$ and $r_{A}(J) \neq(0)$. If, in addition, $r_{A}\left(\ell_{A}(I)\right)=I$ and $\ell_{A}\left(r_{A}(J)\right)=J$, then $A$ is called a dual algebra.

Received by the editors May 5, 1989.

1980 Mathematics Subject Classification (1985 Revision). Primary 46H10; Secondary 46H99.

Key words and phrases. Semisimple Banach algebra, dual algebra, socle, minimal idempotent. 
An idempotent $e$ in a Banach algebra $A$ is said to be minimal if $e A e$ is a division algebra. In case $A$ is semisimple, this is equivalent to saying that $A e$ (resp. $e A$ ) is a minimal left (resp. right) ideal of $A$.

We say that a Banach algebra $A$ has a right approximate identity if there exists a net $\left\{u_{t}\right\}$ in $A$ such that $x=\lim x u_{t}$ for all $x$ in $A .\left\{u_{t}\right\}$ is not necessarily bounded. Analogously we define a left approximate identity.

Notation. If $A$ is a Banach algebra which is a dense subalgebra of a Banach algebra $B$, then we write $\|\cdot\|$ for the norm on $A$ and $|\cdot|$ for the norm on $B$.

In this paper, all algebras and linear spaces under consideration are over the field $C$ of complex numbers.

\section{BANACH ALgEbRA Which is A DENSE SUbALGEbRA IN ANOTHER BANACH ALGEBRA}

In this section, $A$ will be a semisimple Banach algebra which is a dense subalgebra of a semisimple Banach algebra $B$ such that $\|\cdot\|$ majorizes $|\cdot|$ on $A$. Let $E$ be a subset of $A$. Then it is clear that $\ell_{B}(E)=\ell_{B}\left(c l_{A}(E)\right)=\ell_{B}\left(c l_{B}(E)\right)$ and $r_{B}(E)=r_{B}\left(c l_{A}(E)\right)=r_{B}\left(c l_{B}(E)\right)$.

Lemma 3.1. Let $A$ be a dual algebra. Then for any proper closed right ideal $J$ of $A$, we have

$$
J=c l_{B}(J) \cap A=r_{B}\left(\ell_{B}(J)\right) \cap A .
$$

Proof. Since $\ell_{A}(J) \subset \ell_{B}\left(c l_{B}(J)\right), \ell_{B}\left(c l_{B}(J)\right) \neq(0)$ and so $c l_{B}(J) \neq B$. Hence $c l_{B}(J)$ is a proper closed right ideal of $B$ and so

$$
\begin{aligned}
J & \subset c l_{B}(J) \cap A \subset r_{B}\left(\ell_{B}\left(c l_{B}(J)\right)\right) \cap A \\
& =r_{B}\left(\ell_{B}(J)\right) \cap A \subset r_{B}\left(\ell_{A}(J)\right) \cap A \\
& =r_{A}\left(\ell_{A}(J)\right)=J .
\end{aligned}
$$

Therefore $J=c l_{B}(J) \cap A=r_{B}\left(\ell_{B}(J)\right) \cap A$.

Theorem 3.2. Let $A$ be a dual algebra. Then the following statements are equivalent:

(1) $B$ is a dual algebra.

(2) For any $x$ in $B, x \in c l_{B}(x B) \cap c l_{B}(B x)$.

(3) For any proper closed right (left) ideal $R$ of $B, R=c_{B}(R \cap A)$.

Proof. By [11, p. 79, Theorem 3.2], $B$ is an annihilator algebra and $A$ and $B$ have the same socle $S$, which is dense in both $A$ and $B$.

$(1) \Rightarrow(2)$. It follows from [5, p. 105, Corollary (2.8.3)].

(2) $\Rightarrow(3)$. Assume (2). Let $R$ be a proper closed right ideal of $B$ and $x \in R$. Since the socle $S$ is dense in $B$, we have $x=\lim _{n} x y_{n}$ in $|\cdot|$ with $y_{n}$ in $S$. Since $x y_{n} \in S \subset A$, we have $x y_{n} \in R \cap A$. Hence $x \in c l_{B}(R \cap A)$ and so $R \subset c_{B}(R \cap A)$. Therefore $R=\operatorname{cl}_{B}(R \cap A)$. Similarly, we can show that $R=c l_{B}(R \cap A)$, if $R$ is a proper closed left ideal of $B$. Therefore (3) is true.

(3) $\Rightarrow(1)$. Assume (3). Let $R$ be a proper closed right ideal of $B$. Then by [5, p. 98, Corollary (2.8.7)], $R$ is contained in a maximal modular right ideal $M$ 
of $B$. Therefore by [5, p. 97, Theorem (2.8.5)], $r_{B}\left(\ell_{B}(R)\right) \subset r_{B}\left(\ell_{B}(M)\right)=M \neq$ $B$. Hence $r_{B}\left(\ell_{B}(R)\right)$ is a proper closed right ideal of $B$. Let $J=c l_{A}(R \cap A)$. Then $c l_{B}(J)=c l_{B}(R \cap A)=R$. Since $r_{B}\left(\ell_{B}(J)\right)=c l_{B}\left(r_{B}\left(\ell_{B}(J)\right) \cap A\right)$, it follows from Lemma 3.1 that

$$
\begin{aligned}
r_{B}\left(\ell_{B}(R)\right) & =r_{B}\left(\ell_{B}(J)\right)=c l_{B}\left(r_{B}\left(\ell_{B}(J)\right) \cap A\right) \\
& =c l_{B}(J)=R .
\end{aligned}
$$

Similarly, we can show that $\ell_{B}\left(r_{B}(R)\right)=R$, if $R$ is a closed left ideal of $B$. Therefore $B$ is a dual algebra. This completes the proof of the theorem.

Corollary 3.3. Suppose that $B$ has a left approximate identity and a right approximate identity. Then if $A$ is a dual algebra, so is $B$.

Proof. For any $x$ in $B$, it is clear that $x \in c l_{B}(x B) \cap c l_{B}(B x)$. Hence by Theorem 3.2, $B$ is a dual algebra.

Remark 1. Let $A$ be an $A^{*}$-algebra which is a dense subalgebra of a $B^{*}$-algebra $B$. It is well known that if $A$ is a dual algebra, so is $B$. This result also follows from Corollary 3.3 , because $B$ has a bounded approximate identity.

Remark 2. A Banach algebra with an unbounded left approximate identity and unbounded right approximate identity may not have a bounded approximate identity (see [2, p. 487, Example 4.2]).

On the other hand, if $B$ is a dual algebra, $A$ may not be a dual algebra. In fact, $A$ may not be an annihilator algebra (for example, see $[9$, p. 1033] and $[10$, p. 293]).

The following result is useful in the next section.

Theorem 3.4. Let $B$ be a dual algebra. Then the following statements are equivalent:

(1) $A$ is a dual algebra.

(2) For any proper closed right (left) ideal $N$ of $A, N=c l_{B}(N) \cap A$ and for any proper closed right (left) ideal $R$ of $B, R=c l_{B}(R \cap A)$.

Proof. $(1) \Rightarrow(2)$. Assume that $A$ is a dual algebra. Since $B$ is a dual algebra, by Theorem 3.2, $R=c l_{B}(R \cap A)$. Let $N$ be a proper closed right ideal of $A$ and $x \in c l_{B}(N) \cap A$. Then there exists a sequence $\left\{x_{n}\right\} \subset N$ such that $x_{n} \rightarrow x$ in $|\cdot|$. Hence for any minimal idempotent $e$ of $A$, we have $x_{n} e \rightarrow x e$ in $|\cdot|$. Since by $\left[11\right.$, p. 78 , Lemma 3.1], $\|\cdot\|$ and $|\cdot|$ are equivalent on $A e, x_{n} e \rightarrow x e$ in $\|\cdot\|$. Since $x_{n} e \in N, x e \in N$, and so $x e A \subset N$. Since $e$ is arbitrary, it follows that $x S_{A} \subset N$, where $S_{A}$ is the socle of $A$ and so $c_{A}(x A) \subset N$. Therefore, by [5, p. 97, Corollary (2.8.3)], $x \in \mathrm{cl}_{A}(x A) \subset N$. Hence it follows that $c l_{B}(N) \cap A \subset N$ and so $N=c l_{B}(N) \cap A$. A similar statement is true for left ideals. Consequently, (2) is true.

(2) $\Rightarrow(1)$. Suppose that (2) is true. Let $N$ be a proper closed right ideal of $A$. Since $N=c l_{B}(N) \cap A, c l_{B}(N)$ is a proper closed right ideal of $B$. Since $B$ is a dual algebra, $\ell_{B}(N)=\ell_{B}\left(c l_{B}(N)\right) \neq(0)$. Since $\ell_{B}(N)$ is a proper closed 
left ideal of $B$, by $(2), \ell_{B}(N)=c l_{B}\left(\ell_{B}(N) \cap A\right)=c l_{B}\left(\ell_{A}(N)\right)$. In particular, $\ell_{A}(N) \neq(0)$. Also we have

$$
\begin{aligned}
N & =c l_{B}(N) \cap A=r_{B}\left(\ell_{B}\left(c l_{B}(N)\right)\right) \cap A \\
& =r_{B}\left(\ell_{B}(N)\right) \cap A=r_{B}\left(c l_{B}\left(\ell_{A}(N)\right)\right) \cap A \\
& =r_{B}\left(\ell_{A}(N)\right) \cap A=r_{A}\left(\ell_{A}(N)\right) .
\end{aligned}
$$

Similarly, we can show that $J=\ell_{A}\left(r_{A}(J)\right)$ for any closed left ideal $J$ of $A$. Therefore $A$ is a dual algebra and this completes the proof of the theorem.

Corollary 3.5. Assume that, for any proper closed right (left) ideal $R$ of $B$, $R=c l_{B}(R \cap A)$. Then the following statements are equivalent:

(1) $A$ is a dual algebra.

(2) $B$ is a dual algebra and, for any proper closed right (left) ideal $N$ of $A$, $N=c l_{B}(N) \cap A$.

Proof. This follows from Theorems 3.2 and 3.4,

The following result is essentially contained in [6, p. 262, Theorem 4.2].

Theorem 3.6. Let $B$ be a dual algebra. Then the following statements are equivalent:

(1) $A$ is a dual algebra.

(2) $A$ and $B$ have the same socle $S$ that is dense in $A$.

Proof. (1) $\Rightarrow(2)$. Suppose that $A$ is a dual algebra. Then by [11, p. 79, Theorem 3.2], $A$ and $B$ have the same socle that is dense in $A$.

$(2) \Rightarrow(1)$. This follows from [6, p. 262, Theorem 4.2].

\section{BANACH ALGEBRA WHICH IS A DENSE TWO-SIDED IDEAL IN ANOTHER BANACH ALGEBRA}

In this section, $A$ will be a semisimple Banach algebra which is a dense twosided ideal of a semisimple Banach algebra $B$. Then $\|\cdot\|$ majorizes $|\cdot|$ on $A$, there exists a constant $M$ such that

$$
\|a b\| \leq M\|a\||b| \text { and }\|b a\| \leq M\|a\||b|,
$$

for all $a$ in $A$ and $b$ in $B$, and $A$ and $B$ have the same socle (see [11, p. 78, Lemma 2.1] and [1, p. 3]). (In [1], a slip is made in not assuming that $A=B \cdot A$ in Proposition 3.3 and Theorems 3.4 and 4.2.)

Theorem 4.1. Suppose that $B$ has a bounded right (resp. left) approximate identity $\left\{u_{t}\right\}$. Then $A$ has a right (resp. left) approximate identity if and only if $x \in \mathrm{cl}_{A}(x A)$ (resp. $x \in \mathrm{cl}_{A}(A x)$ ) for all $x$ in $A$.

Proof. If $A$ has a right approximate identity, then clearly, $x \in c l_{A}(x A)$ for all $x$ in $A$.

Conversely, suppose that $x \in c_{A}(x A)$ for all $x$ in $A$. By [2, p. 486, Lemma $2.1]$, we can assume that $\left\{u_{t}\right\} \subset A$. We show that $\left\{u_{t}\right\}$ is a right approximate identity of $A$. Since $\left\{u_{t}\right\}$ is bounded in $B$, there exists a constant $K$ such that 
$\left|u_{t}\right| \leq K$ for all $t$. Let $x \in A$. Since $x \in c l_{A}(x A)$, for given $\varepsilon>0$, there exists $y \in A$, such that $\|x-x y\|<\varepsilon / 3 M K(<\varepsilon / 3)$. Since $\left\{u_{t}\right\}$ is a right approximate identity of $B$, there exists $t_{0}$ such that, for $t>t_{0},\left|y-y u_{t}\right|<\varepsilon / 3 M\|x\|$. Therefore,

$$
\begin{aligned}
\left\|x-x u_{t}\right\| & \leq\|x-x y\|+\left\|x y-x y u_{t}\right\|+\left\|x y u_{t}-x u_{t}\right\| \\
& \leq\|x-x y\|+M\|x\|\left|y-y u_{t}\right|+M\|x y-x\|\left|u_{t}\right| \\
& <\varepsilon / 3+\varepsilon / 3+\varepsilon / 3=\varepsilon
\end{aligned}
$$

Therefore $\left\{u_{t}\right\}$ is a right approximate identity of $A$. This completes the proof. Remark. If $A$ has a bounded right (or left) approximate identity, then $A=B$. In fact, suppose that $\left\{u_{t}\right\}$ is a bounded right approximate identity of $A$ with $\left\|u_{t}\right\| \leq K$, where $K$ is a constant. Then, for each $x$ in $A$, we have

$$
\begin{aligned}
\|x\| & \leq\left\|x-e_{t} x\right\|+\left\|e_{t} x\right\| \leq\left\|x-e_{t} x\right\|+M\left\|e_{t}\right\||x| \\
& \leq\left\|x-e_{t} x\right\|+M K|x| .
\end{aligned}
$$

Since $\left\|x-e_{t} x\right\| \rightarrow 0$, it follows that $\|x\| \leq M K|x|$. Therefore $\|\cdot\|$ and $|\cdot|$ are equivalent on $A$ and so $A=B$.

Theorem 4.2. Suppose that $B$ has a bounded right approximate identity and a bounded left approximate identity. Then the following conditions are equivalent:

(1) $A$ is a dual algebra.

(2) $B$ is a dual algebra and $x \in c_{A}(x A) \cap c l_{A}(A x)$ for all $x$ in $A$.

Proof. (1) $\Rightarrow(2)$. Assume that $A$ is a dual algebra. Then by [5, p. 105, Corollary (2.8.3)], $x \in c_{A}(x A) \cap c_{A}(A x)$ for all $x$ in $A$. Since $B$ has a bounded right approximate identity and a bounded left approximate identity, by Theorem $3.2, B$ is a dual algebra.

(2) $\Rightarrow(1)$. Assume that $(2)$ is true. Let $\left\{u_{t}\right\}$ be a bounded right approximate identity of $B$. Then by Lemma 4.1 , we can assume that $\left\{u_{t}\right\}$ is a right approximate identity of $A$. Let $R$ be a closed right ideal of $B$ and $y \in R$. Since $y u_{t} \in R \cap A$ and $y u_{t} \rightarrow y$ in $|\cdot|$, it follows that $y \in c l_{B}(R \cap A)$. Therefore $R \subset c l_{B}(R \cap A)$, and so $R=c_{B}(R \cap A)$. Let $N$ be a closed right ideal of $A$ and $x \in \operatorname{cl}_{B}(N) \cap A$. Write $x=\lim _{n} x_{n}$ in $|\cdot|$ with $x_{n} \in N$. Let $z \in A$. Since $x_{n} z \in N$ and $\left\|x z-x_{n} z\right\| \leq M\left|x-x_{n}\right|\|z\|$, it follows that $x z \in N$; in particular $x u_{t} \in N$ for all $t$. Since $x u_{t} \rightarrow x$ in $\|\cdot\|$, it follows that $x \in N$. Therefore $c l_{B}(N) \cap A \subset N$ and so $N=\operatorname{cl}_{B}(N) \cap A$. A similar statement is true for left ideals. Therefore, by Theorem 3.4, $A$ is a dual algebra. This completes the proof of the theorem.

The following result was proved by Johnson and Lahr (see [3, p. 313, Theorem 2]).

Corollary 4.3. Let $A$ be an $A^{*}$-algebra that is a dense two-sided ideal of a $B^{*}$ algebra $B$. Then $A$ is a dual algebra if and only if $B$ is a dual algebra and $A^{2}$ is dense in $A$. 
Proof. Suppose that $A$ is a dual algebra. By Theorem 4.2, $B$ is a dual algebra. Since the socle of $A$ is dense in $A, A^{2}$ is dense in $A$.

Conversely, suppose that $B$ is a dual algebra and $A^{2}$ is dense in $A$. Then by $\left[3\right.$, p. 312, Theorem 1], $A$ has an approximate identity, and so $x \in c l_{A}(x A) \cap$ $c l_{A}(A x)$ for all $x$ in $A$. Therefore by Theorem 4.2, $A$ is a dual algebra.

\section{REFERENCES}

1. B. A. Barnes, Banach algebras which are ideals in a Banach algebra, Pacific J. Math. 38 (1971), 1-7.

2. P. G. Dixon, Approximate identities in normed algebras, Proc. London Math. Soc. 26 (3) (1973), 485-496.

3. D. L. Johnson and C. D. Lahr, Dual $A^{*}$-algebras of the first kind, Proc. Amer. Math. Soc. 74 (1979), 311-314.

4. T. Ogasawara and K. Yoshinaga, Weakly completely continuous Banach *-algebras, J. Sci. Hiroshima Univ. Ser. A 18 (1954), 15-36.

5. C. E. Rickart, General theory of Banach algebras, University Series in Higher Math, Van Nostrand, Princeton, NJ, 1960.

6. B. J. Tomiuk and B. Yood, Topological algebras with dense socle, J. Funct. Analysis 28 (1978), 254-277.

7. P. K. Wong, Modular annihilator $A^{*}$-algebras, Pacific J. Math. 37 (1971), 825-834.

8. On the Arens product and certain Banach algebras, Trans. Amer. Math. Soc. 180 (1973), 437-448.

9. - The second conjugates of certain Banach algebras, Canad. J. Math. 27 (1975), 1029-1035.

10. $\_$A minimax formula for dual $B^{*}$-algebras, Trans. Amer. Math. Soc. 224 (1976), 281-298.

11. _ A note on annihilator Banach algebras, Bull. Austral. Math. Soc. 38 (1988), 77-81.

12. B. Yood, Ideals in topological rings, Canad. J. Math. 16 (1964), 28-45.

Department of Mathematics, Seton Hall University, South Orange, New Jersey 07079 